\title{
Predictive diagnosis of the cancer prone Li-Fraumeni syndrome by accident: new challenges through whole genome array testing
}

\author{
T Schwarzbraun, ${ }^{1}$ A C Obenauf, ${ }^{1}$ A Langmann, ${ }^{2}$ U Gruber-Sedlmayr, ${ }^{3}$ K Wagner, \\ M R Speicher, ${ }^{1}$ P M Kroisel'
}

${ }^{1}$ Institute of Human Genetics, Medical University of Graz, Graz, Austria; ${ }^{2}$ Department of Ophthalmology, Medical University of Graz, Graz, Austria; ${ }^{3}$ Department of

Neuropediatrics, Medical University of Graz, Austria

Correspondence to:

Professor M R Speicher

Institute of Human Genetics,

Medical University of Graz,

Harrachgasse 21/8, A-8010

Graz, Austria; michael.

speicher@medunigraz.at

TS and ACO contributed equally to this article.

Received 21 November 2008

Revised 8 January 2009

Accepted 27 January 2009

Published Online First

5 March 2009

\section{ABSTRACT}

Background: Li-Fraumeni syndrome greatly increases the risk of developing several types of cancer and is usually caused by TP53 germline mutations. Predictive testing of at-risk family members is only offered after a complex genetic counselling process. Recently the clinical implementation of array comparative genomic hybridisation (CGH) has revolutionised the diagnosis of patients with syndromic or non-syndromic mental retardation and has evolved to a routinely performed high resolution whole genome scan.

Methods and results: When using array CGH to identify the cause for mental retardation in a 7-year-old child we found a submicroscopic de novo deletion of chromosome 17p13.1, which includes several genes likely to be causative for her phenotype, and also of TP53.

Conclusion: Thus, array CGH resulted in an unintended predictive diagnosis of an increased tumour susceptibility as observed in Li-Fraumeni syndrome.

The list of well defined inherited cancer predisposition syndromes, which can be attributed to a hereditary susceptibility and have far reaching implications for all family members, is steadily growing. Many of these syndromes are caused by germline alterations in a tumour suppressor gene. Usually one functional copy of a tumour suppressor gene is sufficient to exert the function. However, inactivation of both alleles by mutation or deletion can result in uncontrolled proliferation and may therefore contribute to tumorigenesis. Hence, individuals who have already one dysfunctional tumour suppressor gene copy in all somatic cells due to a germline mutation may have such an increased tumour risk whenever the function of the second copy is compromised in a cell. This mechanism is usually referred to as Knudson's "two-hit hypothesis". ${ }^{1}$

In familial cancer syndromes the identification of at-risk family members by predictive testing is often recommended as enhanced surveillance for early diagnosis and prevention of disease is a critical part of primary care. Due to the extensive consequences of such predictive testing it is usually only offered after a complex genetic counselling process. Sophisticated surveillance guidelines with proven benefit were developed for several cancer prone syndromes such as Lynch syndrome (hereditary non-polyposis colon cancer (HNPCC)), ${ }^{2}$ hereditary breast and ovarian cancer due to mutations in BRCA1 or BRCA2, ${ }^{3}$ or for hereditary childhood tumours such as retinoblastoma. ${ }^{45}$
Array comparative genomic hybridisation $(\mathrm{CGH})$ has evolved to a standard application in clinical genetics, especially in individuals with syndromic or non-syndromic mental retardation. As a consequence high resolution scans of entire genomes for small gains and losses anywhere in the genome are now conducted routinely. However, the commonly applied execution of array $\mathrm{CGH}$ bears the risk that deletions of genes involved in hereditary cancer syndromes, especially tumour suppressor genes, may also be identified, which would then result in an unintended predictive diagnosis of a cancer prone syndrome. Here we report such a case in which we identified a submicroscopic de novo deletion of chromosome 17p13.1, which included among other genes the tumour suppressor TP53 gene. This scenario represents new challenges for both clinical oncologists and genetic counsellors.

\section{CASE REPORT}

The proband was born at 42 weeks by spontaneous vaginal delivery following an uncomplicated pregnancy; the Apgar score was 7/9/10. Birth weight was $4180 \mathrm{~g}$ (>90th centile), length was $52 \mathrm{~cm}$ (75th centile), and the head circumference (OFC) was $37 \mathrm{~cm}$ (90th centile).

Examinations at 4 and 5 months revealed a psychomotor retardation with a generalised muscle hypotonia. Dysmorphic features included a broad and low set nasal bridge, a short philtrum and a bifid uvula. The child could not establish visual contact. Brain magnetic resonance imaging (MRI) showed the presence of an enlarged fourth ventricle, hypoplasia of the cerebellar vermis and corpus callosum, anomalies usually classified as incomplete manifestation of a Dandy-Walker malformation or Dandy-Walker variant. Within the first year short episodes of myoclonic seizures occurred, which could be prevented by administration of valproic acid and lamotrigine.

At 18 months of age she was able to sit. A severe bilateral visual impairment and a high myopia of $-12 \mathrm{D}$ were diagnosed.

At 7 years of age she was able to walk without support but with ataxic movements. She spoke a few words. At this age the patient was presented to our counselling service. In order to clarify the cause of her impairment we obtained informed consent from the parents to perform cytogenetic analysis and high resolution array $\mathrm{CGH}$.
This paper is freely availat online under the BMJ Journa unlocked scheme, see http:// jmg.bmj.com/info/unlocked.dtl 


\section{METHODS}

\section{Cytogenetic analysis}

Chromosome banding analyses of the proposita and her parents were done according to standard protocols.

\section{Array CGH}

Array CGH was carried out using a whole genome oligonucleotide microarray platform (Human Genome CGH 44B Microarray Kit; Agilent Technologies, Santa Clara, California, USA). This array consists of approximately 43000 60-mer oligonucleotide probes with a spatial resolution of $43 \mathrm{~kb}$. Samples were labelled with the Bioprime Array CGH Genomic Labeling System (Invitrogen, Carlsberg, California, USA) according to the manufacturer's instructions. Further steps were performed according to the manufacturer's protocol (version 6.0). Slides were scanned using a microarray scanner (G2505B) and images were analysed using CGH Analytics software 3.4.40 (both from Agilent Technologies) with the statistical algorithm ADM-2, sensitivity threshold was 6.0. At least three consecutive clones had to be aberrant to be identified as significant change.

\section{Fluorescence in situ hybridisation (FISH)}

FISH was performed with a commercially available probe for the TP53-region (LSI p53 Abbott/Vysis) according to the manufacturer's instructions.

\section{Quantitative real-time polymerase chain reaction (qPCR)}

As previously described, qPCR was performed in order to verify the results from array $\mathrm{CGH}$ and to narrow down the breakpoint region. ${ }^{6}$ After narrowing down the breakpoint region to about $10 \mathrm{~kb}$, we conducted PCR with primers from both sides. The junction fragment was subsequently sequenced and compared with the genomic sequence.

\section{Sequencing}

RNA was isolated from peripheral blood using the PAXgene blood RNA system according to the manufacturer's protocols (PreAnalytiX). For cDNA synthesis we used the Omniscript RT Kit (Qiagen) with oligo dT primers. Monoallelic expression of GUCY2D was determined by sequencing a single nucleotide polymorphism (SNP) (rs2816) from the 3'UTR of GUCY2D in both genomic DNA and cDNA.

Sequencing was performed by cycle sequencing using the ABI BigDye Terminator Cycle Sequencing Kit according to the supplier's protocol and was analysed on an ABI3100 genetic analyser (both ABI).

\section{RESULTS AND DISCUSSION}

Standard banding analysis of the proposita showed a normal female karyotype $(46, X X)$. In a next step we performed array $\mathrm{CGH}$, which revealed a small deletion in chromosome 17p13.1 (fig 1). When analysing the DNA of the parents and their daughter by real-time PCR we identified this deletion only when using the daughter's but not the parental DNA. Provided that none of the parents has a mosaic constellation the deletion most likely occurred de novo. Furthermore, we applied a commercially available TP53-specific probe to metaphase spreads of the proposita, which confirmed the deletion (fig 2). In addition, we determined the deletion size and the localisation of the breakpoints by sequencing (fig 3). In summary, the exact size of the deletion is $774 \mathrm{~kb}$ and contains 47 genes according to current database entries (Ensemble release 50; www.ensembl. org).

Several of the deleted genes can presumably be related to some of the patient's phenotypic features. For example, loss of the KCNAB3 potassium channel gene is likely to be involved in the occurrence of the patient's seizures. Furthermore, the deletion includes several neurotransmitter genes and kinases, which may represent a contributing factor to the mental retardation. A prime example for phenotype-genotype correlation is the GUCY2D gene, which was disrupted from the cisregulatory elements by the breakpoint. We verified monoallelic expression by sequencing an SNP (rs2816) within GUCY2D in both genomic DNA and cDNA. This resulted in cone-rod dystrophy 6 (CORD6; OMIM 601777), a diagnosis which was indeed confirmed by our ophthalmologists and explains our patient's severely impaired vision.

However, we also identified a loss of the TP53 gene within the deleted region. TP53 regulates the cell cycle and functions as a tumour suppressor involved in preventing cancer. As such, TP53 has been designated as the "guardian of the genome". In fact, the TP53 gene is the most commonly mutated gene in human cancer. ${ }^{8}$ The overall lifetime risk of cancer in patients with TP53 germline mutations is in the range of $80-90 \%$, with a risk as high as $40 \%$ within the first two decades of life."

This condition was named Li-Fraumeni syndrome after the physicians who first recognised it. ${ }^{10}$ The classical definition of the Li-Fraumeni syndrome is based on the following three parameters: (1) a proband with a sarcoma diagnosed before the age of 45; (2) a first degree relative with cancer before the age of 45; and (3) another first or second degree relative with either a sarcoma diagnosed at any age or any cancer diagnosed under the age of $45 .{ }^{11}$ Although sarcomas are quite frequently observed in Li-Fraumeni syndrome, a broad range of other tumours may occur including breast cancer, bone cancer, brain tumours, lung cancer, laryngeal cancer, leukaemia, adrenal cortical neoplasia, and others. Furthermore, more relaxed criteria were released for variations of a Li-Fraumeni-like syndrome..$^{12}$

In the absence of a positive family history and without any tumour our patient does not formally fulfil the criteria for LiFraumeni or Li-Fraumeni-like syndrome. However, the complete loss of one copy of the TP53 gene in our patient should result in a similar if not identical tumour risk as affected members of Li-Fraumeni syndrome families according to the aforementioned two-hit hypothesis. Therefore, we assess that our patient has a greatly increased tumour risk with all the unusual characteristics of the Li-Fraumeni syndrome, including that several kinds of cancers may be involved, that cancer may strike at a young age, and that cancer may strike several times throughout the patient's life.

As mentioned before there are cancer prone syndromes in which clear guidelines for surveillance have been established and their benefit has been proven. In contrast, TP53 germline mutations represent a special scenario, as the variable expressivity and penetrance and the diversity of the tumour spectrum render clinical surveillance and genetic testing a difficult task. As a consequence there are no guidelines for patients with TP53 mutations and the benefit of any kind of surveillance has not been proven yet. ${ }^{414}$ Nevertheless, the American Society of Clinical Oncology (ASCO) considers Li-Fraumeni syndrome to be a syndrome for which predictive testing should be considered, especially within research studies. ${ }^{15}$

However, these considerations usually refer to an adult individual who is able to give informed consent. In the case of children, who are not able to give informed consent, predictive 


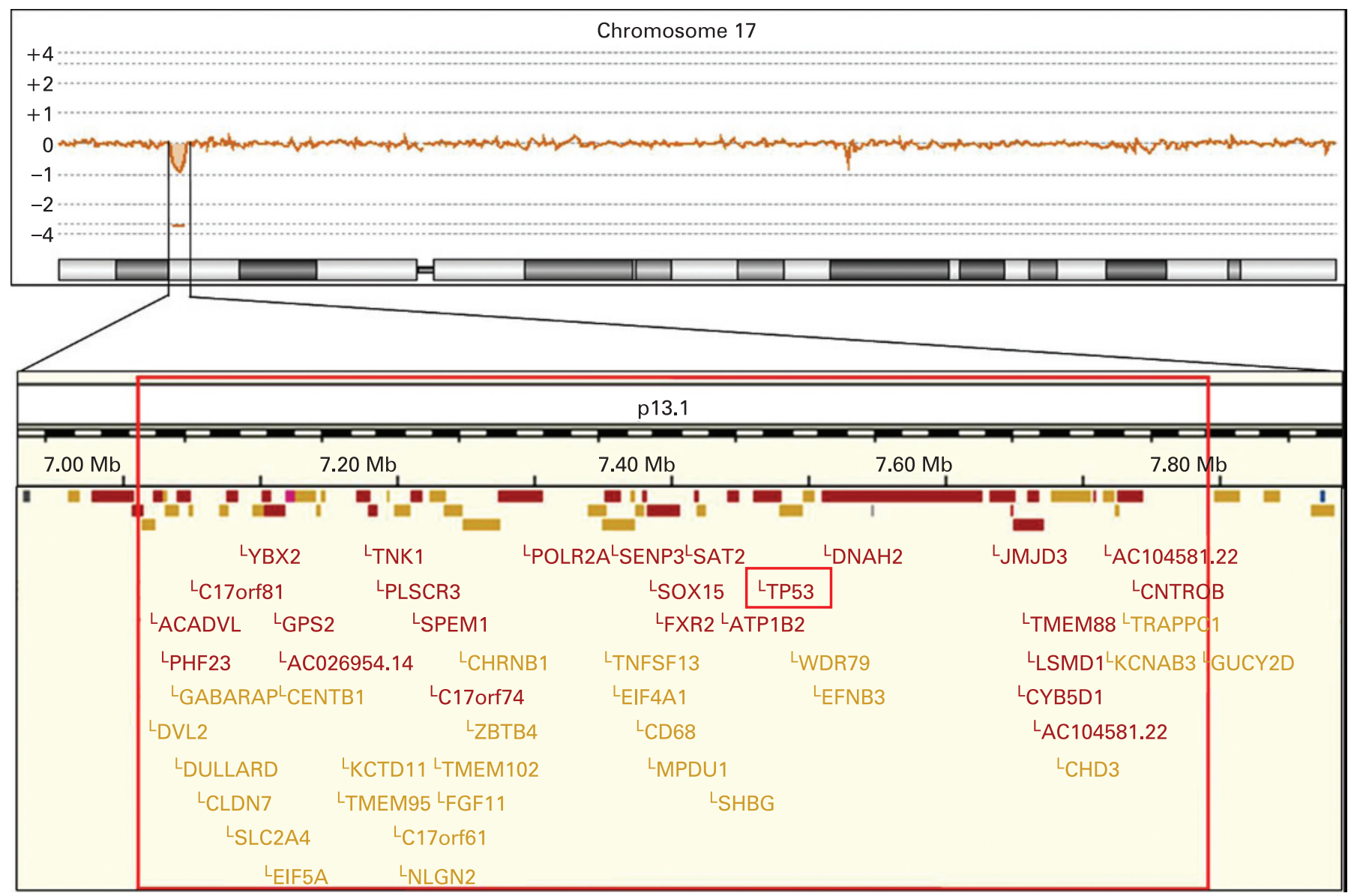

Figure 1 The top panel illustrates the array comparative genomic hybridisation profile of chromosome 17 demonstrating a small deletion in chromosome band 17p13.1. The lower panel depicts an enlargement of the deleted region. The exact localisation of the breakpoints was determined by sequence analysis. The TP53 gene is almost at the centre of the deleted region. The GUCY2D gene is not included in the deleted region. However, the cis-regulatory elements are deleted, which explains the monoallelic expression of the gene and as a consequence the patient's cone-rod dystrophy 6.

TP53 testing is controversial. Recommendations include that a multidisciplinary team should decide whether such a test for a cancer predisposition syndrome, in which many tumours do not manifest until later in life, is to the child's benefit. Therefore, the welfare of the child should always be the major consideration. ${ }^{5}$

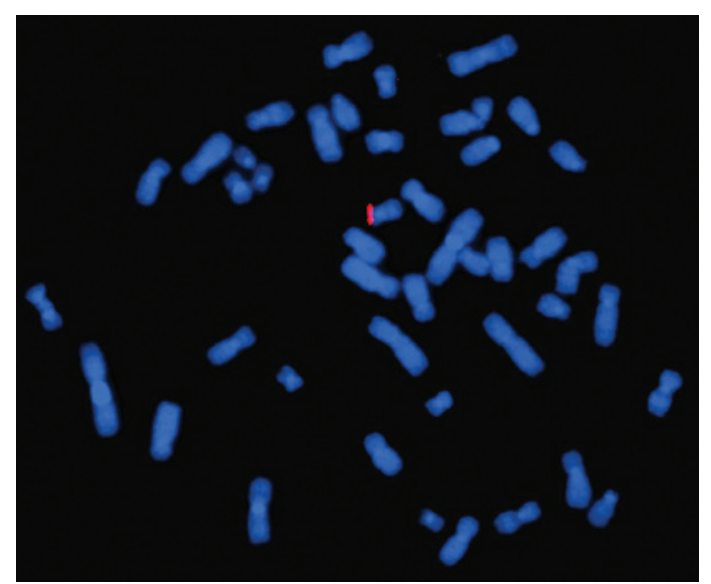

Figure 2 Hybridisation of a commercially available TP53 specific probe to metaphase spreads of the proposita, resulting in only one signal confirming the deletion of the respective region.
In our case such careful considerations before testing had not been possible. The patient's situation is further complicated by her severe mental retardation, as she may complain about possible symptoms from a tumour growth only at an advanced stage or even not at all, which poses an additional risk for a delayed diagnosis. We informed the patient's parents in extensive genetic counselling sessions. Furthermore, we formed a multidisciplinary team in order to discuss possible surveillance options. At present this surveillance is confined to regular

Deletion chr17:7.066.210-7.840.187 Total of 773.978 bp

GCAACCTGCC TCCCATTTCT CCCCTTCTCC TCCGCCCAAT TCCAGGCCCC ACTGCTCCCC GTCCTCCACG CCCTGAATAT CCCAT DEI TACAGAACAG TGTTATCTTT TATGTAAAAA GGGGACTAGA AGAAAATAGA TTCTTATTGC TTGCGTATGC CCAAGGAGCG CCACAAAACG

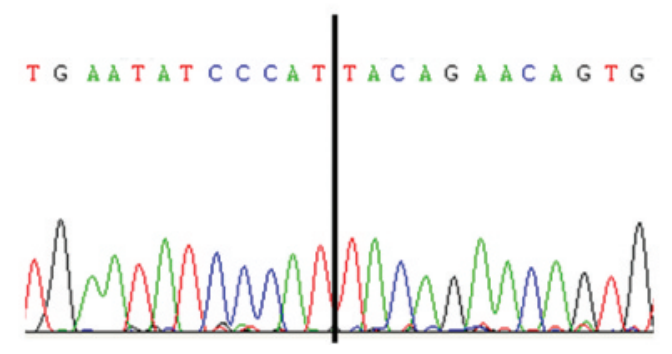

Figure 3 Chromatogram showing the exact location of the breakpoints and the resulting size of the deletion. 
physical examinations and ultrasound examinations of the abdomen.

As array CGH has evolved into a standard diagnostic tool for a variety of conditions, ${ }^{16}$ it is very likely that with the advancing use of this technology similar deletions, or deletions of other cancer prone syndromes, will be identified. In each case it will place the physician or genetic counsellor in a special situation as a risk evaluation for tumour development will have to be offered, although this had not been requested initially by the patient or her/his family. The growing risk of identifying individuals with an increased lifetime risk for cancer by accident will fuel the need for sophisticated risk stratification to achieve a better prediction for the occurrence of possible tumour types and the timing of cancer. ${ }^{17}$

Acknowledgements: We are grateful to Mag. Maria Langer-Winter for critically reading the manuscript.

Funding: Work in our laboratory is supported by the European Commission (DISMAL project, contract no. LSHC-CT-2005-018911; GENINCA project, contract no. 202230) and the FWF (Austrian Science Fund). Anna C. Obenauf is funded by the PhD-Program Molecular Medicine of the Medical University of Graz.

Competing interests: None.

Patient consent: Obtained.

\section{REFERENCES}

1. Knudson AG Jr. Mutation and cancer: statistical study of retinoblastoma. Proc Natl Acad Sci USA 1971:68:820-3.

2. Vasen HF, Möslein G, Alonso A, Bernstein I, Bertario L, Blanco I, Burn J, Capella G, Engel C, Frayling I, Friedl W, Hes FJ, Hodgson S, Mecklin JP, Møller P, Nagengast F, Parc Y, Renkonen-Sinisalo L, Sampson JR, Stormorken A, Wijnen J. Guidelines for the clinical management of Lynch syndrome (hereditary non-polyposis cancer). J Med Genet 2007;44:353-62.
3. Horsman D, Wilson BJ, Avard D, Meschino WS, Kim Sing C, Plante M, Eisen A, Howley HE, Simard J, National Hereditary Cancer Task Force. Clinical management recommendations for surveillance and risk-reduction strategies for hereditary breast and ovarian cancer among individuals carrying a deleterious BRCA1 or BRCA2 mutation. J Obstet Gynaecol Can 2007;29:45-60.

4. Field M, Shanley S, Kirk J. Inherited cancer susceptibility syndromes in paediatric practice. J Paediatr Child Health 2007:43:219-29.

5. Rao A, Rothman J, Nichols KE. Genetic testing and tumor surveillance for children with cancer predisposition syndromes. Curr Opin Pediatr 2008;20:1-7.

6. Schwarzbraun T, Ullmann R, Schubert $M$, Ledinegg $M$, Ofner $L$, Windpassinger $C$, Wagner K, Kroisel PM, Petek E. Characterization of a de novo complex chromosome rearrangement (CCR) involving chromosomes 2 and 12, associated with mental retardation and impaired speech development. Cytogenet Genome Res 2006;115:84-9.

7. Lane DP. Cancer. p53, guardian of the genome. Nature 1992;358:15-16.

8. Friend S. p53: a glimpse at the puppet behind the shadow play. Science 1994;265:334-5.

9. Le Bihan C, Moutou C, Brugières L, Feunteun J, Bonaïti-Pellié C. ARCAD: a method for estimating age-dependent disease risk associated with mutation carrier status from family data. Genet Epidemiol 1995;12:13-25.

10. Li FP, Fraumeni JF. Rhabdomyosarcoma in children: an epidemiologic study and identification of a familial cancer syndrome. J Nat Cancer Inst 1969;43:1364-73.

11. Li FP, Fraumeni JF Jr, Mulvihill JJ, Blattner WA, Dreyfus MG, Tucker MA, Miller RW A cancer family syndrome in twenty-four kindreds. Cancer Res 1988:48:5358-62.

12. Birch JM, Hartley AL, Tricker KJ, Prosser J, Condie A, Kelsey AM, Harris M, Jones $\mathrm{PH}$, Binchy A, Crowther D, Craft AW, Eden OB, Evans DGR, Thompson E, Mann JR, Martin J, Mitchell ELD, Santibánez-Koref MF. Prevalence and diversity of constitutional mutations in the p53 gene among 21 Li-Fraumeni families. Cancer Res 1994; 54:1298-304.

13. Eeles RA. Germline mutations in the TP53 gene. Cancer Surv 1995;25:101-24.

14. Strahm B, Malkin D. Hereditary cancer predisposition in children: genetic basis and clinical implications. Int J Cancer 2006;119:2001-6.

15. American Society of Clinical Oncology. American Society of Clinical Oncology policy statement update: genetic testing for cancer susceptibility. J Clin Oncol 2003:21:2397-406

16. Speicher MR, Carter NP. The new cytogenetics: blurring the boundaries with molecular biology. Nat Rev Genet 2005;6:782-92.

17. Tabori U, Malkin D. Risk stratification in cancer predisposition syndromes: lessons learned from novel molecular developments in Li-Fraumeni syndrome. Cancer Res 2008:68:2053-7.

\section{Access a vast information database with Toll-Free linking}

"Toll-free" linking gives you immediate access to the full text of many of the cited articles in a paper's reference list-FOR FREE. With the support of HighWire's vast journal catalogue, a huge reference library is now open to you. If HighWire hosts the journal, you can view the full text of the referenced article, completely free of charge by following the Free Full Text links in the references. 